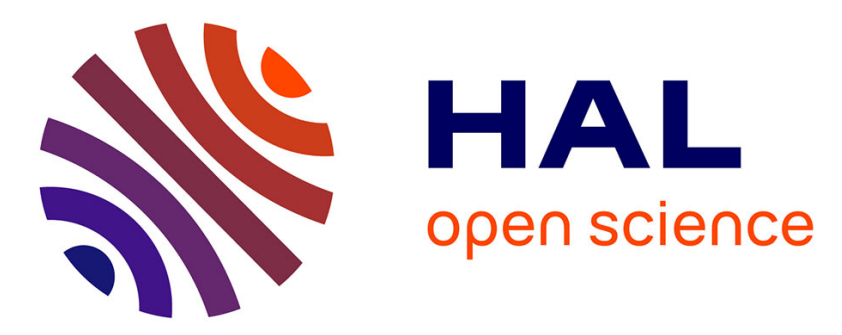

\title{
SINC APPROXIMATION OF THE HEAT FLUX ON THE BOUNDARY OF A TWO-DIMENSIONAL FINITE SLAB
}

Pham Hoang Quan, Dang Duc Trong, Alain Pham Ngoc Dinh

\section{> To cite this version:}

Pham Hoang Quan, Dang Duc Trong, Alain Pham Ngoc Dinh. SINC APPROXIMATION OF THE HEAT FLUX ON THE BOUNDARY OF A TWO-DIMENSIONAL FINITE SLAB. Numerical Functional Analysis and Optimization, 2006, 27 (5-6), pp.685-695. hal-00008986

\section{HAL Id: hal-00008986 https://hal.science/hal-00008986}

Submitted on 26 Sep 2005

HAL is a multi-disciplinary open access archive for the deposit and dissemination of scientific research documents, whether they are published or not. The documents may come from teaching and research institutions in France or abroad, or from public or private research centers.
L'archive ouverte pluridisciplinaire HAL, est destinée au dépôt et à la diffusion de documents scientifiques de niveau recherche, publiés ou non, émanant des établissements d'enseignement et de recherche français ou étrangers, des laboratoires publics ou privés. 


\title{
SINC APPROXIMATION OF THE HEAT FLUX ON THE BOUNDARY OF A TWO-DIMENSIONAL FINITE SLAB
}

\author{
by \\ P. H. Quan, D. D. Trong \\ HoChiMinh City National University, Department of Mathematics and Informatics \\ 227 Nguyen Van Cu, Q5, HoChiMinh City, Vietnam \\ Email: tquan@pmail.vnn.vn \\ $\&$ \\ P. N. Dinh Alain \\ Mathematics Department, Mapmo UMR 6628, BP 67-59 \\ 45067, Orleans cedex, France \\ Email: alain.pham@univ-orleans.fr
}

\begin{abstract}
We consider the two-dimensional problem of recovering globally in time the heat flux on the surface of a layer inside of a heat conducting body from two interior temperature measurements. The problem is ill-posed. The approximation function is represented by a twodimensional Sinc series and the error estimate is given.
\end{abstract}

Key words and phrases : heat equation, heat flux, Sinc series, ill-posed problem, regularization, numerical results. AMS Subjects Classification : 35K05, 35K20, 35R30, 42A38

Address for correspondence: Alain Pham Ngoc Dinh

\section{Introduction}

In this paper, we consider the problem of recovering the heat flux on the surface of a thin layer inside of a heat conducting body from transient temperature measurements. The problem is raised in many applications in Physics and Geology. In fact, in many physical situation (see, e.g., [B]), we cannot attach a temperature sensor at the surface of the body (for example, the skin of a missile). On the other hand, we can easily measure the temperature history at an interior point of the body. Hence, to get the heating history in the body, we want to use temperature measured in the interior of the body. In the simplest model, the heat-conducting body is assumed to have a constant conductivity and represented by the half-line $x>0$ (see, e.g. [C, EM, LN, TV]),. While giving many useful results, this model is not suitable for the case of a body having a series of superposed layers, each of which has a constant conductivity.

Precisely, we shall consider the problem corresponding to a thin layer of the body represented by the strip $\mathbb{R} \times(0,3)$, say. Let $u$ be the temperature in the strip. For the uniqueness of solution, we shall have to measure the temperature history at two interior lines $\mathbb{R} \times\{y=1\}$ and $\mathbb{R} \times\{y=2\}$. From these measurements, we can identify uniquely the heating history inside of the layer (see, e.g., $[\mathrm{B}])$. However, to make clearly ideas, we limit to the problem of finding the heat flux $u_{y}(x, 1, t) \equiv$ $w(x, t)$ on the one side of the boundary of the slab $\mathbb{R} \times(1,2)$. Our problem is classical and can be 
changed to the one of finding the solution of a convolution equation of Volterra type which can be solved on any finite time interval by the iteration (see, e.g., [F]). However, it is surprised that the problem is ill-posed if we consider the problem on the global time interval and the literature on this way is very scarce. In fact, despite uniqueness, the global solution in $L^{2}\left(\mathbb{R} \times \mathbb{R}_{+}\right)$is unstability and hence, in this point of view, a sort of regularization is in order.

As discussed in the latter paragraph, the main purpose of our paper is to present a regularization of the problem. Moreover, an effective way of approximating the heat flux $w$ is also worthy of considering. Indeed, we recall that the problem can be changed into a convolution equation. There are many methods for regularizing the equation (see [TA, B]). In the present paper, using the method of truncated integration, one can approximate the Fourier transform of the solution by a function having the compact support in $\mathbb{R}^{2}$. Therefore, the solution can be represented by an expansion of two dimensional Sinc series (see [AGTV]). The Sinc method is based on the Cardinal functions

$$
S(p, d)(z)=\frac{\sin [(\pi(z-p d)) / d]}{\pi(z-p d) / d}, p \in \mathbb{Z}, d>0
$$

which dates back to the works of many mathematicians (Bohr, de la Vallee Poussin, E. T. Whittaker, ...). The one dimensional version of the method is studied very clearly and completely in [S]. Some primary results related to the two dimensional Sinc approximation were given in [AGTV]. As is

known, the Sinc series converges very rapidly at an incredible $0\left(e^{-c n^{1 / 2}}\right)$ rate, where $c>0$ and $n$ is the dimension of approximation (see [S]). Hence, this method, which is new in our knowledge, is very effective.

The remainder of the present paper is divided into three sections. In Section 2, we state precisely the problem, change it into an integral equation of convolution type, and state the main result of our paper. In Section 3, we give the proof of the main result and in Section 4 we present some numerical results in terms of two dimensional Sinc functions.

\section{Notations and the main result}

Consider the problem of determining the heat flux

$$
u_{y}(x, 1, t) \equiv w(x, t), x \in \mathbb{R}, t>0,
$$

where $u$ satisfies

$$
\Delta u-\frac{\partial u}{\partial t}=0 \quad x \in \mathbb{R}, 1<y<2, t>0,
$$

with the boundary conditions

$$
\begin{aligned}
& u(x, 2, t)=g(x, t), x \in \mathbb{R}, t>0, \\
& u(x, 1, t)=f(x, t), x \in \mathbb{R}, t>0,
\end{aligned}
$$


and the initial condition

$$
u(x, y, 0)=0, x \in \mathbb{R}, 1<y<2
$$

where $f, g$ are given.

We shall transform Problem (1)-(5) into a convolution equation.

Put

$$
\Gamma(x, y, t, \xi, \eta, \tau)=\frac{1}{4 \pi(t-\tau)} \exp \left(-\frac{(x-\xi)^{2}+(y-\eta)^{2}}{4(t-\tau)}\right)
$$

and

$$
G(x, y, t, \xi, \eta, \tau)=\Gamma(x, y, t, \xi, \eta, \tau)-\Gamma(x, 4-y, t, \xi, \eta, \tau) .
$$

We have

$$
G_{\xi \xi}+G_{\eta \eta}+G_{\tau}=0 .
$$

Integrating the identity

$$
\operatorname{div}(G \nabla u-u \nabla G)-\frac{\partial}{\partial \tau}(u G)=0
$$

over the domain $(-n, n) \times(1,2) \times(0, t-\varepsilon)$ and letting $n \rightarrow \infty, \varepsilon \rightarrow 0$, we have

$$
\begin{aligned}
\int_{-\infty}^{+\infty} \int_{0}^{t} g(\xi, \tau) G_{\eta}(x, y, t, \xi, 2, \tau) d \xi d \tau & +\int_{-\infty}^{+\infty} \int_{0}^{t} G(x, y, t, \xi, 1, \tau) w(\xi, \tau) d \xi d \tau \\
& -\int_{-\infty}^{+\infty} \int_{0}^{t} f(\xi, \tau) G_{\eta}(x, y, t, \xi, 1, \tau) d \xi d \tau+u(x, y, t)=0 .
\end{aligned}
$$

Hence

$$
\begin{aligned}
& \int_{-\infty}^{+\infty} \int_{0}^{t} G(x, y, t, \xi, 1, \tau) w(\xi, \tau) d \xi d \tau=-u(x, y, t)+ \\
& \int_{-\infty}^{+\infty} \int_{0}^{t} G_{\eta}(x, y, t, \xi, 1, \tau) f(\xi, \tau) d \xi d \tau-\int_{-\infty}^{+\infty} \int_{0}^{t} g(\xi, \tau) G_{\eta}(x, y, t, \xi, 2, \tau) d \xi d \tau .
\end{aligned}
$$

Letting $y \rightarrow 1^{+}$in (8), we have

$$
\int_{-\infty}^{+\infty} \int_{0}^{t}\left[\frac{1}{2 \pi(t-\tau)} \exp \left(-\frac{(x-\xi)^{2}}{4(t-\tau)}\right)-\frac{1}{2 \pi(t-\tau)} \exp \left(-\frac{(x-\xi)^{2}+4}{4(t-\tau)}\right)\right] w(\xi, \tau) d \xi d \tau
$$




$$
\begin{aligned}
& =-f(x, t)-\frac{1}{2 \pi} \int_{-\infty}^{+\infty} \int_{0}^{t} \frac{1}{(t-\tau)^{2}} \exp \left(-\frac{(x-\xi)^{2}+4}{4(t-\tau)}\right) f(\xi, \tau) d \xi d \tau \\
& +\frac{1}{2 \pi} \int_{-\infty}^{+\infty} \int_{0}^{t} g(\xi, \tau) \frac{1}{(t-\tau)^{2}} \exp \left(-\frac{(x-\xi)^{2}+1}{4(t-\tau)}\right) d \xi d \tau .
\end{aligned}
$$

This implies that

$$
(P-Q) * w(x, t)=-f(x, t)-R_{1} * f(x, t)+R_{2} * g(x, t)
$$

where we put $w(x, t)=f(x, t)=g(x, t)=0$ if $(x, t) \in \mathbb{R} \times(-\infty, 0)$,

$$
\begin{gathered}
P(x, t)=\left\{\begin{array}{cc}
\frac{1}{t} \exp \left(-\frac{x^{2}}{4 t}\right) & (x, t) \in \mathbb{R} \times[0,+\infty) \\
0 & (x, t) \in \mathbb{R} \times(-\infty, 0)
\end{array},\right. \\
Q(x, t)=\left\{\begin{array}{cc}
\frac{1}{t} \exp \left(-\frac{x^{2}+4}{4 t}\right) & (x, t) \in \mathbb{R} \times[0,+\infty) \\
0 & (x, t) \in \mathbb{R} \times(-\infty, 0)
\end{array},\right. \\
R_{1}(x, t)=\left\{\begin{array}{cc}
\frac{1}{t^{2}} \exp \left(-\frac{x^{2}+4}{4 t}\right) & (x, t) \in \mathbb{R} \times[0,+\infty) \\
0 & (x, t) \in \mathbb{R} \times(-\infty, 0)
\end{array}\right.
\end{gathered}
$$

and

$$
R_{2}(x, t)=\left\{\begin{array}{cr}
\frac{1}{t^{2}} \exp \left(-\frac{x^{2}+1}{4 t}\right) & (x, t) \in \mathbb{R} \times[0,+\infty) \\
0 & (x, t) \in \mathbb{R} \times(-\infty, 0)
\end{array} .\right.
$$

From now on, every function $\varphi=\varphi(x, t)$ defined on $\mathbb{R} \times \mathbb{R}_{+}$will be seen as a function defined on $\mathbb{R}^{2}$ by putting $\varphi(x, t)=0$ for $t<0$.

Now, we state our main result.

\section{Theorem}

Let $a \in(0,2)$ and $\varepsilon \in\left(0,2^{-3 / 2}\right)$. Assume that $w_{0} \in L^{2}\left(\mathbb{R}^{2}\right)$ is the (unique) solution of (9) corresponding to the exact data $f_{0}, g_{0} \in L^{2}\left(\mathbb{R}^{2}\right)$ and that $f, g \in L^{2}\left(\mathbb{R}^{2}\right)$ are measured data satisfying

$$
\mid f-f_{0}\left\|_{2} \leq \varepsilon,\right\| g-g_{0} \|_{2} \leq \varepsilon
$$

where $\|.\|_{2}$ is the $L^{2}\left(\mathbb{R}^{2}\right)$-norm.

Then, from $f, g$, we can construct a sequence $\left\{a_{m n, \varepsilon}\right\}(m, n \in \mathbb{Z})$ and a $C>0$ independent of E such that

$$
\left\|w_{0}-\sum_{n=-\infty}^{+\infty} \sum_{|m| \leq|n|} a_{m n, \varepsilon} S\left(m, \pi b_{\varepsilon}^{2}\right)(x) S\left(n, \pi b_{\varepsilon}^{2}\right)(t)\right\|_{2} \leq \sqrt{C \varepsilon^{2-a}+\eta(\varepsilon)}
$$


where $b_{\varepsilon}=\varepsilon^{a / 4}$,

$$
S(p, d)(z)=\frac{\sin [\pi(z-p d) / d]}{\pi(z-p d) / d}, p \in \mathbb{Z}, d>0,
$$

and $\eta(\varepsilon) \rightarrow 0$ as $\varepsilon \downarrow 0$.

Moreover, if we assume in addition that $w_{0} \in L^{1}\left(\mathbb{R}^{2}\right) \cap H^{2}\left(\mathbb{R}^{2}\right)$ then, for $a=\frac{4}{3}$ there exists a $D>0$ independent of $\varepsilon$ such that

$$
\left\|w_{0}-\sum_{n=-\infty}^{+\infty} \sum_{|m| \leq|n|} a_{m n, \varepsilon} S\left(m, \pi b_{\varepsilon}^{2}\right)(x) S\left(n, \pi b_{\varepsilon}^{2}\right)(t)\right\|_{2}<D \varepsilon^{\frac{1}{3}} .
$$

\section{Proof}

The proof consists of two steps. In Step 1, we shall construct a regularized solution and in Step 2, we give the error estimate.

\section{Step 1 : Construction of a regularized solution}

We have

$$
\begin{aligned}
\hat{P}(z, r) & =\frac{1}{2 \pi} \int_{-\infty}^{+\infty} \int_{-\infty}^{+\infty} P(x, t) e^{-i(x z+t r)} d x d t \\
& =\frac{1}{\sqrt{2} \sqrt{z^{4}+r^{2}}}\left[\sqrt{\sqrt{z^{4}+r^{2}}+z^{2}}-i \operatorname{sgn}(r) \sqrt{\sqrt{z^{4}+r^{2}}-z^{2}}\right]
\end{aligned}
$$

and

$$
\begin{aligned}
\hat{Q}(z, r) & =\frac{1}{2 \pi} \int_{-\infty}^{+\infty} \int_{-\infty}^{+\infty} Q(x, t) \epsilon^{-i(x z+t r)} d x d t \\
& =\frac{1}{\sqrt{z^{4}+r^{2}}} e^{-2 u}[u \cos 2 v-v \sin 2 v-i \operatorname{sgn}(r)(u \sin 2 v+v \cos 2 v)]
\end{aligned}
$$

where

$$
u=\frac{1}{\sqrt{2}} \sqrt{\sqrt{z^{4}+r^{2}}+z^{2}}, v=\frac{1}{\sqrt{2}} \sqrt{\sqrt{z^{4}+r^{2}}-z^{2}} .
$$

We put

$$
\begin{aligned}
F(x, t) & =-f(x, t)+R_{1} * f(x, t)+R_{2} * g(x, t), \\
F_{0}(x, t) & =-f_{0}(x, t)+R_{1} * f_{0}(x, t)+R_{2} * g_{0}(x, t) .
\end{aligned}
$$

Taking the Fourier-transform of both sides of (9), we have

$$
(\hat{P}(z, r)-\hat{Q}(z, r)) \hat{w}(z, r)=\hat{F}(z, r) .
$$


It call for the following formula of the regularized equation

$$
w_{\varepsilon}(x, t)=\frac{1}{2 \pi} \int_{D_{\varepsilon}} \frac{\hat{F}(z, r)}{\hat{P}(z, r)-\hat{Q}(z, r)} e^{i(x z+t r)} d z d r .
$$

where

$$
b_{\varepsilon}=\varepsilon^{\frac{a}{5}} \in(0,1), D_{\varepsilon}=\left\{(z, r) / b_{\varepsilon}^{4} \leq z^{4}+r^{2} \leq \frac{1}{b_{\varepsilon}^{4}}\right\} .
$$

We have

$$
\text { supp } \hat{w}_{\varepsilon} \subset D_{\varepsilon} \subset\left[-\frac{1}{b_{\varepsilon}^{2}}, \frac{1}{b_{\varepsilon}^{2}}\right] \times\left[-\frac{1}{b_{\varepsilon}^{2}}, \frac{1}{b_{\varepsilon}^{2}}\right] .
$$

As in [AGLT], p. 121, we have

$$
\begin{aligned}
w_{\varepsilon}(x, t) & =\sum_{n=-\infty}^{+\infty} \sum_{|m| \leq|n|} w_{\varepsilon}\left(m \pi b_{\varepsilon}^{2}, n \pi b_{\varepsilon}^{2}\right) S\left(m, \pi b_{\varepsilon}^{2}\right)(x) S\left(n, \pi b_{\varepsilon}^{2}\right)(t) \\
& =\sum_{n=-\infty}^{+\infty} \sum_{|m| \leq|n|} a_{m n} S\left(m, \pi b_{\varepsilon}^{2}\right)(x) S\left(n, \pi b_{\varepsilon}^{2}\right)(t)
\end{aligned}
$$

where

$$
a_{m n}=w_{\varepsilon}\left(m \pi b_{\varepsilon}^{2}, n \pi b_{\varepsilon}^{2}\right)=\frac{1}{2 \pi} \int_{D_{\varepsilon}} \frac{\hat{F}(z, r)}{\hat{P}(z, r)-\hat{Q}(z, r)} e^{i(m z+n r) \pi b_{\varepsilon}^{2}} d z d r .
$$

\section{Step 2 : Error estimates}

Noting that

$$
(\hat{P}(z, r)-\hat{Q}(z, r)) \widehat{w}_{0}(z, r)=\widehat{F}_{0}(z, r)
$$

we have

$$
\left\|w_{0}-w_{\varepsilon}\right\|_{2}^{2}=\left|\widehat{w}_{0}-\widehat{w}_{\varepsilon}\right|_{2}=\int_{D_{\varepsilon}}\left|\frac{\hat{F}(z, r)-\hat{F}_{0}(z, r)}{\hat{P}(z, r)-\hat{Q}(z, r)}\right|^{2} d z d r+\int_{\mathbb{R}^{2} \backslash D_{\varepsilon}}\left|\hat{w}_{0}(z, r)\right|^{2} d z d r .
$$

For $(z, r) \in D_{\varepsilon}$, we have

$$
\begin{aligned}
|\hat{P}(z, r)-\hat{Q}(z, r)| & =\frac{1}{\sqrt{u^{2}+v^{2}}} \sqrt{1-2 e^{-2 u} \cos 2 v+e^{-4 u}} \\
& \geq \frac{1}{\sqrt[4]{z^{4}+r^{2}}}\left(1-e^{-\sqrt{2} \sqrt{\sqrt{z^{4}+r^{2}}+z^{2}}}\right) \\
& \geq b_{\varepsilon}\left(1-\epsilon^{-\sqrt{2} b_{\varepsilon}}\right) \geq \frac{1-\epsilon^{-\sqrt{2}}}{\sqrt{2}} b_{\varepsilon}^{2} .
\end{aligned}
$$


On the other hand,

$$
\begin{aligned}
\left\|\hat{F}-\hat{F}_{0}\right\|_{2} & =\left\|F-F_{0}\right\|_{2} \\
& \leq\left[|| R_{1} \|_{1}+1\right]\left\|f-f_{0}\right\|_{2}+\left\|R_{2}\right\|_{1}\left\|g-g_{0}\right\|_{2} \\
& \leq\left(\left\|R_{1}\right\|_{1}+|| R_{2} \|_{1}+1\right) \varepsilon .
\end{aligned}
$$

From (11), (12) and the latter inequality, we get

$$
\left\|w_{\varepsilon}-w_{0}\right\|_{2}^{2} \leq \frac{2 \varepsilon^{2}\left(\left\|R_{1}\right\|_{1}+|| R_{2} \|_{1}+1\right)^{2}}{\left(1-e^{-\sqrt{2}}\right)^{2} b_{\varepsilon}^{4}}+\eta(\varepsilon)=\frac{2\left(\left\|R_{1}\right\|_{1}+\left\|R_{2}\right\|_{1}+1\right)^{2}}{\left(1-e^{-\sqrt{2}}\right)^{2}} \varepsilon^{2-a}+\eta(\varepsilon)
$$

where $\eta(\varepsilon)=\int_{\mathbb{R}^{2} \backslash D_{\varepsilon}}\left|\hat{w}_{0}(z, r)\right|^{2} d z d r$.

Moreover, if $w_{0} \in H^{2}\left(\mathbb{R}^{2}\right)$, then choosing $b_{\varepsilon}=\varepsilon^{\frac{1}{3}}<\frac{1}{\sqrt{2}}$.

We have

$$
\left\|w_{0}-w_{\varepsilon}\right\|_{2}^{2} \leq \frac{2\left(\left\|R_{1}\right\|_{1}+\left\|R_{2}\right\|_{1}+1\right)^{2}}{\left(1-e^{-\sqrt{2}}\right)^{2} b_{\varepsilon}^{4}} \varepsilon^{2}+\int_{z^{4}+r^{2}<b_{\varepsilon}^{4}}\left|\hat{w}_{0}(z, r)\right|^{2} d z d r+\int_{z^{4}+r^{2}>\frac{1}{b_{\varepsilon}^{4}}}\left|\hat{w}_{0}(z, r)\right|^{2} d z d r
$$

Note that if $b_{\varepsilon}<\frac{1}{2}$ and $z^{4}+r^{2}<b_{\varepsilon}^{4}$, then $z^{2}+r^{2}<b_{\varepsilon}^{2} \sqrt{2}$, hence

$$
\int_{z^{4}+r^{2}<b_{\varepsilon}^{2}}\left|\hat{w}_{0}(z, r)\right|^{2} d z d r \leq 2|| w_{0} \|_{1}^{2} \pi b_{\varepsilon}^{2}
$$

On the other hand if $b_{\varepsilon}<\frac{1}{2}$ and $z^{4}+r^{2}>\frac{1}{b_{\varepsilon}^{4}}$, then $z^{2}+r^{2}>\frac{1}{b_{\varepsilon}^{2}}$, therefore

$$
\int_{z^{4}+r^{2}>\frac{1}{b_{\varepsilon}^{4}}}\left|\hat{w}_{0}(z, r)\right|^{2} d z d r \leq \int_{z^{4}+r^{2}>\frac{1}{b_{\varepsilon}^{4}}} \frac{\left(z^{2}+r^{2}\right)^{2}\left|\hat{w}_{0}(z, r)\right|^{2}}{\left(z^{2}+r^{2}\right)^{2}} d z d r \leq b_{\varepsilon}^{4} E
$$

where $E=\left\|\left(z^{2}+r^{2}\right) \hat{w}_{0}(z, r)\right\|_{2}^{2}$.

By (13)-(15), we have

$$
\left\|w_{0}-w_{\varepsilon}\right\|_{2}^{2} \leq \frac{2\left(\left\|R_{1}\right\|_{1}+\left\|R_{2}\right\|_{1}+1\right)^{2}}{\left(1-e^{-\sqrt{2}}\right)^{2} b_{\varepsilon}^{4}} \varepsilon^{2}+\left(2\left\|w_{0}\right\|_{1}^{2} \pi+E\right) b_{\varepsilon}^{2} \leq D^{2} \varepsilon^{\frac{2}{3}} .
$$

where

$$
D=\max \left\{\frac{\sqrt{2}\left(\left\|R_{1}\right\|_{1}+\left\|R_{2}\right\|_{1}+1\right)}{1-e^{-\sqrt{2}}}, \sqrt{2\left\|w_{0}\right\|_{1}^{2} \pi+E}\right\} .
$$

The proof of theorem is completed. 


\section{Numerical results}

We present some results of numerical comparison of the regularized representation of the solution given by (10) and the corresponding exact solution of the problem.

Let the problem

$$
\begin{gathered}
\Delta u-\frac{\partial u}{\partial t}=0, \quad(x, y) \in \mathbb{R} \times(0,3), t>0 \\
u(x, 1, t)=\frac{1}{t} \mathrm{e}^{\frac{-x^{2}-1}{4 t}} ; u(x, 2, t)=\frac{1}{t} \mathrm{e}^{\frac{-x^{2}-4}{4 t}} ; u(x, y, 0=0
\end{gathered}
$$

whose the unknown is

$$
w(x, t)=u_{y}(x, 1, t)
$$

The exact solution of this problem is

$$
w(x, t)=\frac{-1}{2 t^{2}} \mathrm{e}^{\frac{-x^{2}-1}{4 t}} .
$$

The approximated solution is calculated from the expansion of two-dimensional Sinc series given by (10) in which

$$
\begin{aligned}
& \hat{F}=\frac{\mathrm{e}^{-u}}{u^{2}+v^{2}}[(\alpha u-\beta v]-i(\alpha v+\beta u)]+\frac{\varepsilon}{\pi \sqrt{r^{2}+1} \sqrt{z^{2}+1}} \\
& \alpha=\mathrm{e}^{-2 u} \cos 3 v-\cos v ; \beta=\mathrm{e}^{-2 u} \sin 3 v-\sin v \\
& \hat{P}-\hat{Q}=a_{1}+i b_{1} \text { where } \\
& a_{1}=u-\mathrm{e}^{-2 u}(u \cos 2 v-v \sin v) ; b_{1}=\operatorname{sgn}(r)\left[-v+\mathrm{e}^{-2 u}(v \cos 2 v+u \sin 2 v)\right] .
\end{aligned}
$$

Thus we have

$$
\left\|\hat{F}-\hat{F}_{0}\right\|_{L^{2}\left(\mathbb{R}^{2}\right)}=\varepsilon
$$

which is a perturbation similar to the one operated on the two given functions $f$ and $g$.

With $\varepsilon=\frac{1}{50}, N=50$ (the size of the double series) and for $(x, t) \in[0,2] \times[0,2]$ we have drawn the corresponding approximate surface solution $(x, t) \longrightarrow w_{\varepsilon}(x, t)$ in Fig.1.

To calculate the double integral in (10) we have used the rectangle rule which gives good accuracy if one integrates on the interval $[\varepsilon, 1 / \varepsilon] \times[\varepsilon, 1 / \varepsilon]$. The time of calculus with a good computer is very long: 2 hours for 900 points $M=(x, t) \in[0,2] \times[0,2]$. It is the reason for which we are limited ourselves to a relatively small size of the double series $(N=20)$. For comparison in Fig.2 we have drawn the exact solution $(x, t) \longrightarrow w(x, t)$. 
approximate solution

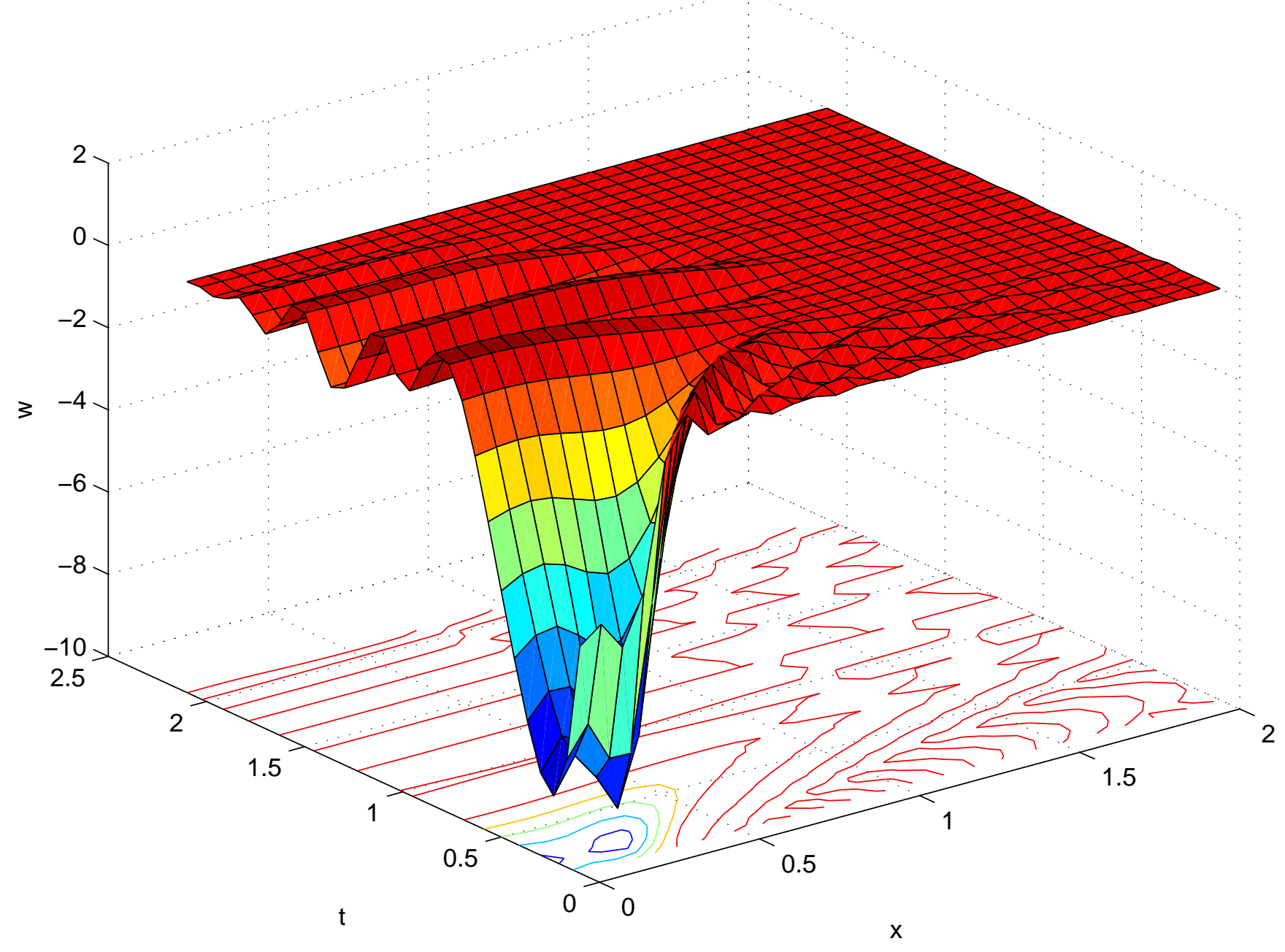

Fig.1 


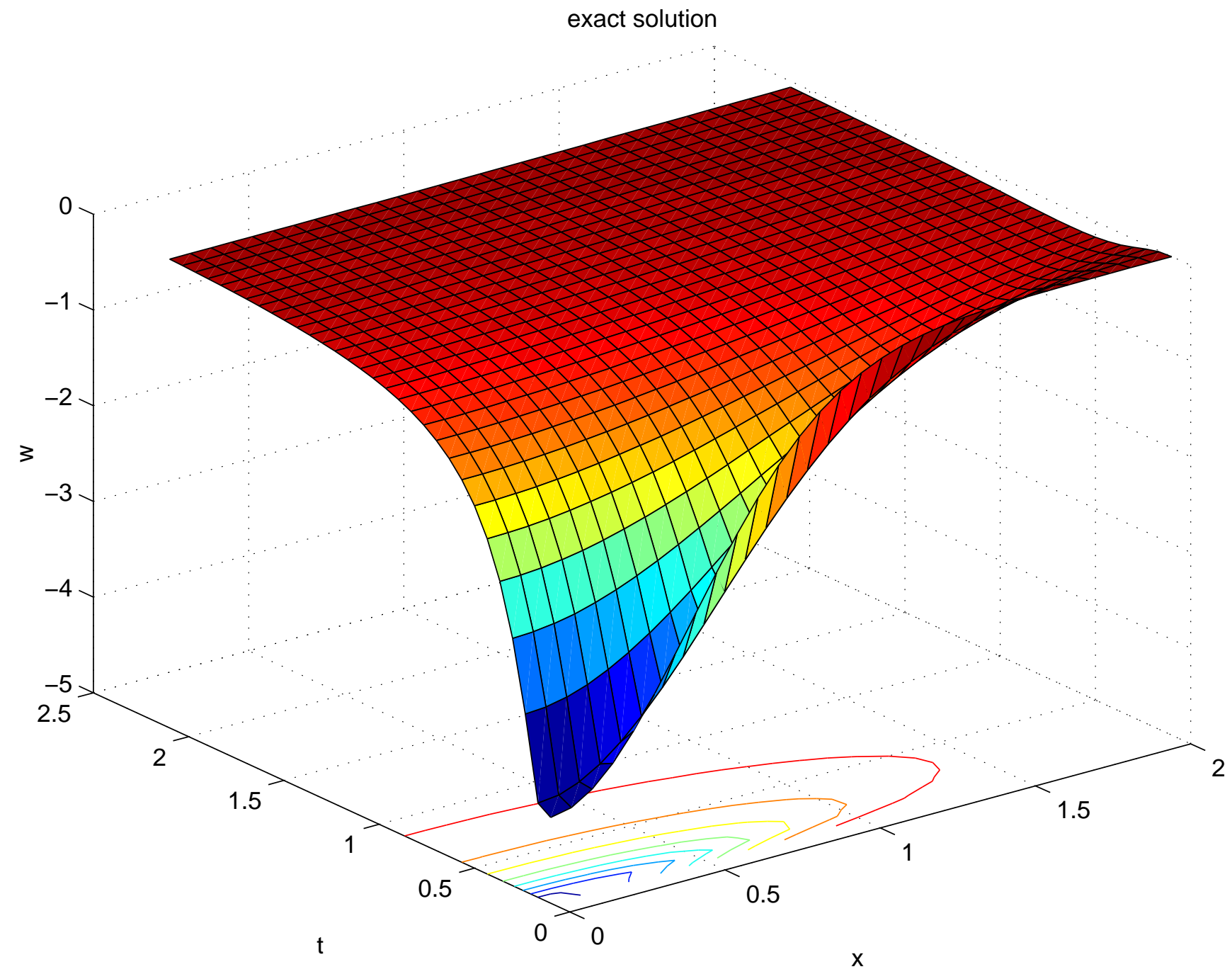

Fig.2

Using the same method as previously we have drawn in Fig. 3 the surface $(x, t) \in[0,4] \times[0,4]$ $w_{\varepsilon}(x, t)$ which is the regularization of the following problem

$$
\begin{gathered}
\Delta u-\frac{\partial u}{\partial t}=0, \quad(x, y) \in \mathbb{R} \times(0,3), t>0 \\
u(x, 1, t)=0 ; u(x, 2, t)=\frac{1}{t} \mathrm{e}^{\frac{-x^{2}-4}{4 t}}\left(1-\mathrm{e}^{\frac{-1}{t}}\right) ; u(x, y, 0=0
\end{gathered}
$$

the unknown being $w(x, t)=u_{y}(x, 1, t)$. The exact solution

$$
w(x, t)=\frac{1}{t^{2}} \mathrm{e}^{\frac{-x^{2}-1}{4 t}} .
$$

is represented in Fig.4. 
approximate solution

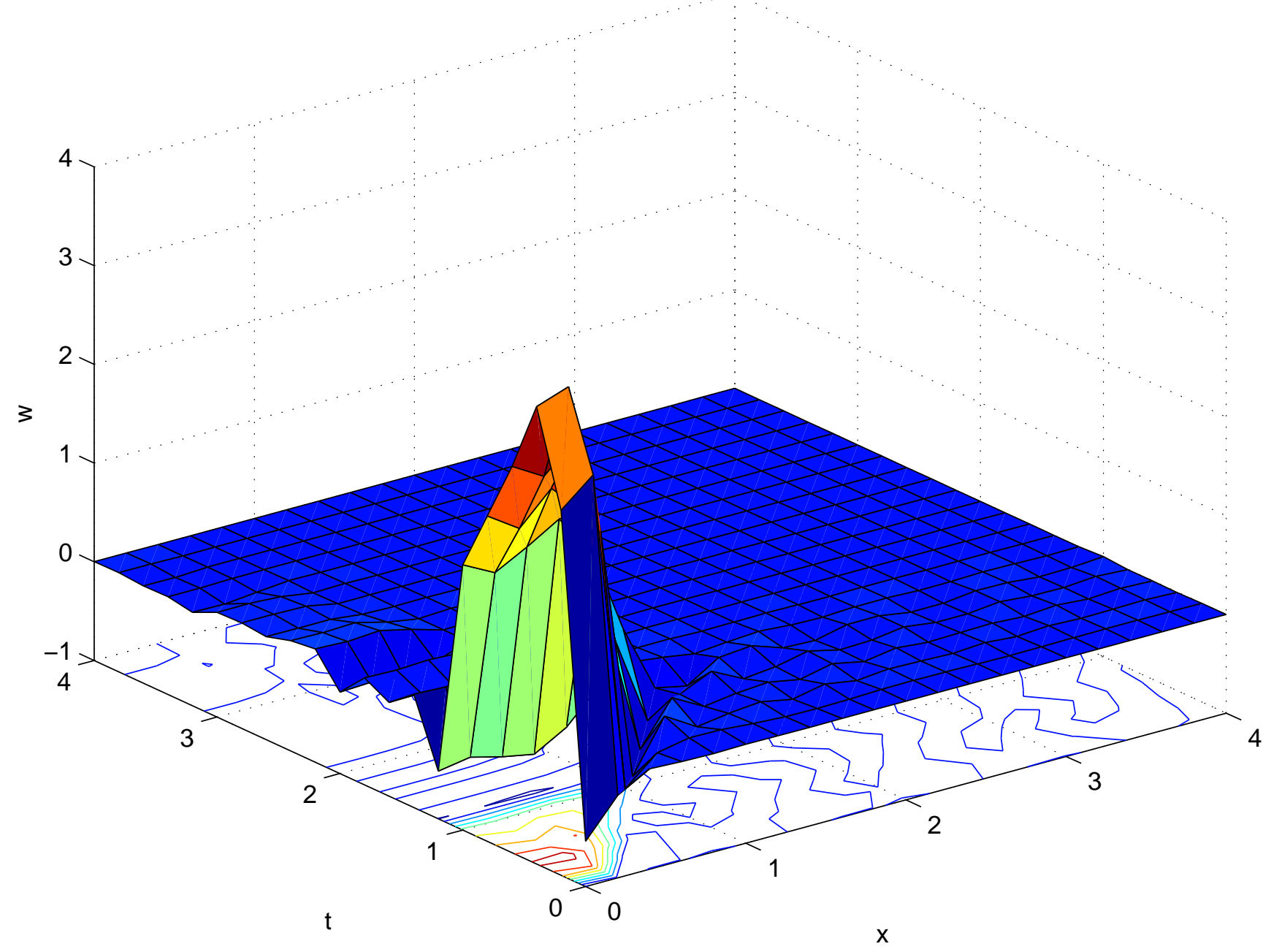

Fig.3 
exact solution

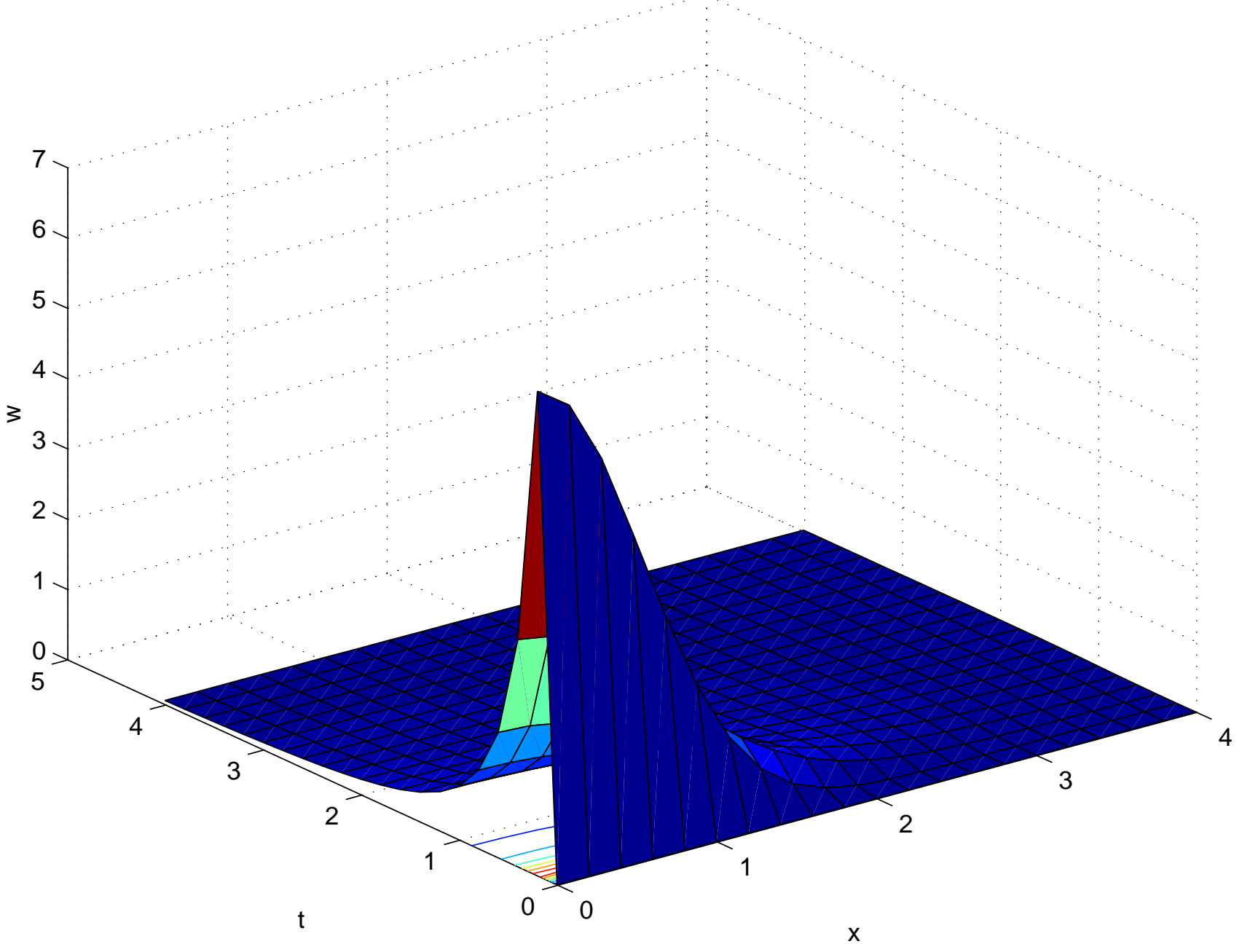

Fig.4 


\section{REFERENCES}

[AGVT] D. D. Ang, R. Gorenflo, L. K. Vy and D. D. Trong. Moment theory and some Inverse Problems in Potential Theory and Heat Conduction, Lecture Notes in Mathematics 1792, Springer, (2002).

[B] J. V. Beck, B. Blackwell and C. R. St. Clair, Jr., Inverse Heat Conduction, Ill-posed Problem, Wiley, New York, (1985).

[C] A. Carasso, Determining surface temperatures from interior observations, SIAM J. Appl. Math. 42 (1981), 558-547.

[E] Erdelyi et al., Tables of Integral Transforms, Vol. 1, Mc Graw-Hill, New York, (1954).

[EM] H. Engl and P. Manselli, Stability estimates and regularization for an inverse heat conduction problem, Numer. Funct. Anal. and Optim. 10 (1989), 517-540.

[F] A. Friedman, Partial Differential Equations of Parabolic Type, Englewood Cliff., N. J., (1964).

[LN] T. T. Le and M. P. Navarro, Surface Temperature From Borehole Measurements : Regularization and Error Estimates, Internl. J. Math and Math Sci. (1995).

[S] Stenger Fr., Numerical methods based on Sinc and analytic functions, Springer Verlag, Berlin - Heidelberg - New York, (1993).

[TA] A. N. Tikhonov and V. Y. Arsenin, Solutions of ill-posed problems, Winston, Washington, (1977).

[TV] G. Talenti and S. Vessella, Note on an ill-posed problem for the heat equation, J Austral. Math. Soc. 32 (1981), 358-368. 